\title{
CONSTRAINED MOTION UPON A SURFACE UNDER A GENERALIZED FIELD OF FORCE
}

\author{
JOHN DECICCO
}

1. Introduction. We shall present here some theorems concerning the trajectories of a particle moving in a given smooth surface when acted upon only by a generalized field of force and free of the pressure of the surface.

Kasner has developed the differential geometry of the dynamical trajectories of general positional fields of force on a plane, in space, and on a surface, in his Princeton Colloquium Lectures. ${ }^{1}$ Recently Kasner and DeCicco have introduced the concept of generalized fields of force which depend not only upon the position of the point but also upon the direction through the point. Positional fields may be described as isotropic and generalized fields as anisotropic. ${ }^{2}$

Consider a generalized field of force defined in a certain region of ordinary space. ${ }^{3}$ Let $\Sigma$ be a smooth surface in this particular region. The trajectories described by a particle constrained to move in $\Sigma$ and acted upon only by this generalized field of force are $\infty^{3}$ in number, just as is the case for a plane.

In general curvilinear coordinates $(u, v)$ of $\Sigma$, these trajectories are defined by a differential equation of the form

$$
v^{\prime \prime \prime}=F\left(u, v, v^{\prime}\right)+G\left(u, v, v^{\prime}\right) v^{\prime \prime}+H\left(u, v, v^{\prime}\right) v^{\prime \prime 2} .
$$

Conversely any differential equation of this form which is satisfied by the totality of geodesics of $\Sigma$ represents the trajectories of a particle constrained to move in $\Sigma$, which is acted upon only by a generalized field of force.

Kasner has proved that in the $(u, v)$-plane, the curves defined by a differential equation of third order of the form (F) may be characterized in the following manner. ${ }^{4}$ The locus of the foci of the osculating parabolas constructed to the $\infty^{1}$ trajectories passing

Presented to the Society, November 2, 1946; received by the editors September 27, 1946.

${ }^{1}$ Kasner, Differential-geometric aspects of dynamics, Amer. Math. Soc. Colloquium Publications, vol. 3, 1913, 1934 (referred to as the Princeton Colloquium). Also see series of papers in Trans. Amer. Math. Soc. vols. 7-11 (1906-1910).

${ }^{2}$ Kasner and DeCicco, A generalized theory of dynamical trajectories, Trans. Amer. Math. Soc. vol. 54 (1943) pp. 23-38.

${ }^{3}$ Kasner and DeCicco, Generalized dynamical trajectories in space, Duke Math. J. vol. 4 (1943) pp. 733-742.

${ }^{4}$ See the Princeton Colloquium, pp. 104-107. 
through a given lineal-element is a special quartic curve.

If the special quartic curve degenerates into a circle passing through the point of the given lineal-element, then $\Sigma$ is a developable surface, which is represented on the $(u, v)$-plane by an unrolling of $\Sigma$ followed by an affinity. In this event, the trajectories are represented as those of a positional field of force in the $(u, v)$-plane.

More generally, if the motion on the surface $\Sigma$ is pictured in the $(u, v)$-plane by a motion due to a positional field together with a resistance depending on the velocity components and acting in the direction of the motion, then $\Sigma$ is of constant curvature and the representation is geodesic.

For any generalized field of force on a surface $\Sigma$, the $\infty^{1}$ trajectories starting from a given lineal-element of $\Sigma$ have osculating spheres at the common point, whose centers lie on a general conic in the plane normal to the element. This conic passes through the center of curvature of the normal section of the surface $\Sigma$ determined by the given lineal-element. If the element is in one of the principal directions of the surface $\Sigma$, the conic touches the normal to $\Sigma$.

Although Kasner first obtained the preceding property for a positional field of force, it is found to be valid also for generalized fields.

After discussing the differential equation of third order defining generalized dynamical trajectories on the surface $\Sigma$, we shall compare the $\infty^{1}$ lines of force and the $\infty^{2}$ rest trajectories. Let a particle which is initially at rest start from a point $O$. The rest trajectory will be tangent to the line of force at $O$. We study the ratio $\rho$ of the departure of the rest trajectory to that of the line of force at $O$. In the positional case, Kasner showed that $\rho=1 / 3$ in the case of first order contact. For the generalized case of the plane, Kasner and DeCicco obtained a more general formula. In the present paper, we shall prove that this more general formula is valid also for a surface $\Sigma$.

2. The acceleration vector. Let a particle be constrained to move in a surface $\Sigma$ which is defined parametrically by the equations

$$
x=x(u, v), \quad y=y(u, v), \quad z=z(u, v) .
$$

Of course, at least two of these three functions of $(u, v)$, are functionally independent in a certain region of the $(u, v)$-plane.

The rectangular components of the velocity vector are given by the expressions

$$
\dot{x}=x_{u} \dot{u}+x_{v} \dot{v}, \quad \dot{y}=y_{u} \dot{u}+y_{v} \dot{v}, \quad \dot{z}=z_{u} \dot{u}+z_{v} \dot{v} .
$$

The speed $\omega$ is defined by the formula 


$$
\omega^{2}=E \dot{u}^{2}+2 F \dot{u} \dot{v}+G \dot{v}^{2} .
$$

The rectangular components of the acceleration vector are

$$
\begin{aligned}
& \ddot{x}=x_{u} \ddot{u}+x_{v} \ddot{v}+x_{u u} \dot{u}^{2}+2 x_{u v} \dot{u} \dot{v}+x_{v v} \dot{v}^{2}, \\
& \ddot{y}=y_{u} \ddot{u}+y_{v} \ddot{v}+y_{u u} \dot{u}^{2}+2 y_{u v} \dot{u} \dot{v}+y_{v v} \dot{v}^{2}, \\
& \ddot{z}=z_{u} \ddot{u}+z_{v} \ddot{v}+z_{u u} \dot{u}^{2}+2 z_{u v} \dot{u} \dot{v}+z_{v v} \dot{v}^{2} .
\end{aligned}
$$

The length $a_{m}$ of the projection of the acceleration vector along any direction $(\delta u, \delta v)$ on $\Sigma$ is found to satisfy the equation

$$
\begin{aligned}
{\left[E \delta u^{2}+\right.} & \left.2 F \delta u \delta v+G \delta v^{2}\right]^{1 / 2} a_{m} \\
= & {\left[E \ddot{u}+F \ddot{v}+\frac{E_{u}}{2} \dot{u}^{2}+E_{v} \dot{u} v+\left(F_{v}-\frac{G_{u}}{2}\right) \dot{v}^{2}\right] \delta u } \\
& +\left[F \ddot{u}+G \ddot{v}+\left(F_{u}-\frac{E_{v}}{2}\right) \dot{u}^{2}+G_{u} \dot{u} \dot{v}+\frac{G_{v}}{2} \dot{v}^{2}\right] \delta v .
\end{aligned}
$$

Before proceeding further with our work, let us recall that the geodesic curvature $k$ of a curve on the surface $\Sigma$ is

$$
k=\frac{H\left[v^{\prime \prime}-n v^{\prime 3}-(2 m-\nu) v^{\prime 2}-(l-2 \mu) v^{\prime}+\lambda\right]}{\left[E+2 F v^{\prime}+G v^{\prime 2}\right]^{3 / 2}},
$$

where

$$
\begin{array}{ll}
l=\frac{1}{2 H^{2}}\left(G E_{u}-2 F F_{u}+F E_{v}\right), \lambda=\frac{1}{2 H^{2}}\left(2 E E_{u}-E E_{v}-F E_{u}\right), \\
m=\frac{1}{2 H^{2}}\left(G E_{v}-F G_{u}\right), & \mu=\frac{1}{2 H^{2}}\left(E G_{u}-F E_{v}\right), \\
n=\frac{1}{2 H^{2}}\left(2 G F_{v}-G G_{u}-F G_{v}\right), & \nu=\frac{1}{2 H^{2}}\left(E G_{v}-2 F F_{v}+F G_{u}\right) .
\end{array}
$$

Of course, $H=\left(E G-F^{2}\right)^{1 / 2}$.

Let $a_{1}$ denote the component of the acceleration vector parallel to the direction defined by $F \delta u+G \delta v=0$, and let $a_{2}$ denote the component of the acceleration vector parallel to the direction defined by $E \delta u+F \delta v=0$. By (5) and (7), we obtain

$$
\begin{aligned}
& a_{1}=\frac{1}{G^{1 / 2} H}\left[\ddot{u}+l \dot{u}^{2}+2 m \dot{u} \dot{v}+n \dot{v}^{2}\right], \\
& a_{2}=\frac{1}{E^{1 / 2} H}\left[\ddot{v}+\lambda \dot{u}^{2}+2 \mu \dot{u} \dot{v}+\nu \dot{v}^{2}\right] .
\end{aligned}
$$


If $a_{t}$ is the tangential component and $a_{n}$ the normal component on the surface $\Sigma$ of the acceleration vector, then from (5) and (6) can be deduced the well known formulas

$$
a_{t}=\dot{v}=v \frac{d v}{d s}, \quad a_{n}=k v^{2}=\frac{v^{2}}{r} .
$$

3. The differential equations of the generalized dynamical trajectories on the surface $\Sigma$. Let $G^{-1 / 2} H^{-1} \phi\left(u, v, v^{\prime}\right)$ denote the component of the generalized force vector parallel to the direction defined by $F \delta u+G \delta v=0$, and let $E^{-1 / 2} H^{-1} \psi\left(u, v, v^{\prime}\right)$ denote the component of the force vector parallel to the direction defined by $E \delta u+F \delta v=0$. By (8), it is found that the differential equations of motion of a particle of unit mass, constrained to move in the surface $\Sigma$ and acted upon only by a generalized field of force, are $e^{5}$

$$
\ddot{u}=\phi-l \dot{u}^{2}-2 m \dot{u} \dot{v}-n \dot{v}^{2}, \ddot{v}=\psi-\lambda \dot{u}^{2}-2 \mu \dot{u} \dot{v}-\nu \dot{v}^{2} .
$$

It is desirable to find $\phi$ and $\psi$ in terms of the original force vector defined by the rectangular components $X\left(u, v, v^{\prime}\right), Y\left(u, v, v^{\prime}\right)$, $Z\left(u, v, v^{\prime}\right)$. The values are

$$
\begin{aligned}
& \phi=G\left(X x_{u}+Y y_{u}+Z z_{u}\right)-F\left(X x_{v}+Y y_{v}+Z z_{v}\right), \\
& \psi=-F\left(X x_{u}+Y y_{u}+Z z_{u}\right)+E\left(X x_{v}+Y y_{v}+Z z_{v}\right) .
\end{aligned}
$$

Substituting (10) into (5) where $\delta u: \delta v=\dot{u}: \dot{v}$, we find that the tangential component $T$ of the force vector along a trajectory is

$$
T=\frac{(E \phi+F \psi)+(F \phi+G \psi) v^{\prime}}{\left(E+2 F v^{\prime}+G v^{\prime 2}\right)^{1 / 2}} .
$$

Substituting the normal direction $\delta v / \delta u=-\left(E+F v^{\prime}\right) /\left(F+G v^{\prime}\right)$ into (5), we discover the normal component $N$ of the force vector to be

$$
N=\frac{H\left(\psi-v^{\prime} \phi\right)}{\left(E+2 F v^{\prime}+G v^{\prime 2}\right)^{1 / 2}} .
$$

From (6), (9), and (13), it is seen that the square of the speed $\omega$ is

$$
\omega^{2}=\frac{\left(\psi-v^{\prime} \phi\right)\left(E+2 F v^{\prime}+G v^{\prime 2}\right)}{v^{\prime \prime}-n v^{\prime 3}-(2 m-\nu) v^{\prime 2}-(l-2 \mu) v^{\prime}+\lambda} .
$$

5 These could have been easily obtained by means of the Lagrangian equations. However, we prefer to derive the differential equations of motion from fundamental principles. For an analogous discussion, see Whittaker, Analytical dynamics, Dover Publications, 1944, pp. 99-109. 
The direction of the projected force vector on the surface $\Sigma$ is the one which yields the maximum value of $T$ in (12). For this, it is necessary and sufficient that $N=0$. Thus the lines of force on the surface $\Sigma$ (if they exist) are given by the differential equation of first order

$$
\psi\left(u, v, v^{\prime}\right)-v^{\prime} \phi\left(u, v, v^{\prime}\right)=0 .
$$

Substituting this into (12), the magnitude $a$ of the projected force vector is

$$
a^{2}=E \phi^{2}+2 F \phi \psi+G \psi^{2} .
$$

From (12), (13), and (16), we find

$$
a^{2}=T^{2}+N^{2} \text {. }
$$

We proceed to eliminate the time $t$ from the equations (10) representing the trajectories on the surface $\Sigma$. For this purpose, it is found useful to introduce the quantity $\Delta$ defined by the equation

$$
\Delta=v^{\prime \prime}-n v^{\prime 3}-(2 m-\nu) v^{\prime 2}-(l-2 \mu) v^{\prime}+\lambda .
$$

Thus $\Delta=0$ only along the geodesics of $\Sigma$.

Upon substituting (10) into the formulas

$$
\dot{v}=v^{\prime} \dot{u}, \quad \ddot{v}=v^{\prime \prime} \dot{u}^{2}+v^{\prime} \ddot{u},
$$

we find

$$
\dot{u}^{2}=\left(\psi-v^{\prime} \phi\right) / \Delta .
$$

Differentiating this with respect to $u$ and eliminating $\dot{u}$, we find the equation of third order

$$
\begin{aligned}
\left(\psi-v^{\prime} \phi\right)\left[\frac{d \Delta}{d u}-2 \Delta\left(l+2 m v^{\prime}\right.\right. & \left.\left.+n v^{\prime 2}\right)\right] \\
& =-2 \phi \Delta^{2}+\Delta \frac{d}{d u}\left(\psi-v^{\prime} \phi\right) .
\end{aligned}
$$

Thus the differential equation of third order representing the $\infty^{3} d y$ namical trajectories on the surface $\Sigma$ is

$$
\begin{aligned}
(\psi- & \left.v^{\prime} \phi\right) \frac{d \Delta}{d u}=\left(\psi_{v^{\prime}}-v^{\prime} \phi_{v^{\prime}}-3 \phi\right) \Delta^{2} \\
& +\left[\left(\psi_{v^{\prime}}-v^{\prime} \phi_{v^{\prime}}-\phi\right)\left\{n v^{\prime 3}+(2 m-\nu) v^{\prime 2}+(l-2 \mu) v^{\prime}-\lambda\right\}\right. \\
& +2\left(\psi-v^{\prime} \phi\right)\left(l+2 m v^{\prime}+n v^{\prime 2}\right) \\
& \left.+\psi_{u}+v^{\prime}\left(\psi_{v}-\phi_{u}\right)-v^{\prime 2} \phi_{v}\right] \Delta .
\end{aligned}
$$


TheOREM 1. Any differential equation of third order of the form

$$
\frac{d \Delta}{d u}=G\left(u, v, v^{\prime}\right) \Delta+H\left(u, v, v^{\prime}\right) \Delta^{2},
$$

where $\Delta$ is given by (18), represents a dynamical family of trajectories of a generalized field of force, constrained to move on a surface $\Sigma$.

The remarkable similarity between this differential equation and that of the type $(G)$ which has been studied extensively by Kasner should be noticed. Of course, this reduces to the G-type when $\Sigma$ is developable and the mapping on the $(u, v)$-plane is an unrolling of $\Sigma$ followed by an affinity.

In the case of a positional field of force, the functions $G$ and $H$ appearing in (23) must be restricted further.

We prove the above theorem. First note that there is a one-to-one correspondence between the functions $(N, T)$ and the functions $(\phi, \psi)$. This correspondence is given by (12) and (13).

Let $P$ and $Q$ be defined by the expressions

$$
P=\frac{2 T}{\left(E+2 F v^{\prime}+G v^{\prime 2}\right)^{1 / 2}}, \quad Q=\frac{N}{H}\left(E+2 F v^{\prime}+G v^{\prime 2}\right)^{1 / 2} .
$$

Thus if $(\phi, \psi)$ are known, the quantities $(P, Q)$ can be calculated, and conversely.

If we use the equations (9) and (24), it is found that the differential equation of the dynamical family may be written in the form

$$
P=\frac{d}{d u}\left(\frac{Q}{\Delta}\right)
$$

The expansion of this is

$$
\frac{d \Delta}{d u}=\left(Q_{u}+v^{\prime} Q_{v}+v^{\prime \prime} Q_{v^{\prime}}\right) \frac{\Delta}{Q}-\frac{P \Delta^{2}}{Q} .
$$

Now $\left(Q_{u}+v^{\prime} Q_{v}\right) / Q$ can represent any function of $\left(u, v, v^{\prime}\right)$ say $R\left(u, v, v^{\prime}\right)$. Finally let

$$
\begin{aligned}
& G=R+\frac{Q_{v^{\prime}}}{Q}\left[n v^{\prime 3}+(2 m-\nu) v^{2}+(l-2 \mu) v^{\prime}-\lambda\right], \\
& H=\frac{Q_{v^{\prime}}-P}{Q} .
\end{aligned}
$$

From these equations, it is seen that $G$ and $H$ can be any pair of 
arbitrary functions of $\left(u, v, v^{\prime}\right)$.

Substituting (27) into (26) and making use of (18), we obtain the equation (23). This completes our proof of Theorem 1.

THEOREM 2. In the auxiliary $(u, v)$-plane, any differential equation of third order of the type (F) which is satisfied by the $\infty^{2}$ geodesics of the surface $\Sigma$ can represent the dynamical trajectories on $\Sigma$ when acted upon only by a generalized field of force.

This result is an immediate consequence of Theorem 1. For upon expanding the differential equation (23) by means of (18), we find the type (F). This must be satisfied by the $\infty^{2}$ geodesics of $\Sigma$ since $\Delta=0$ is a solution of (23). Conversely, if $\Delta=0$ is a solution of a differential equation of third order of the type $(F)$, then it must be written in the form (23). Thus Theorem 2 follows.

4. The generalization upon a surface $\Sigma$ of Kasner's theorem concerning one-third of the curvatures. In this section, we shall study, in the motion of a particle constrained to move on a surface $\Sigma$ when acted upon only by a generalized field of force, the ratio $\rho$ of the departure of the rest trajectory to that of the line of force at a given point $O$. For the positional case, Kasner showed that $\rho=1 / 3$. In the generalized type on a plane, ${ }^{6}$ Kasner and DeCicco obtained a more general formula for $\rho$ in terms of the angular rate $\lambda$. We shall show that this more general formula is valid also upon a surface $\Sigma .^{7}$

At this point, we shall define the angular rate $\lambda$ which is associated to any lineal-element $E$ on the surface $\Sigma$ for the motion of a particle constrained to move on $\Sigma$ when acted upon by a generalized field of force. As the lineal-element $E$ rotates about its point $O$, the projection $F$ on $\Sigma$ of the corresponding force vector also rotates about $O$. The angular rate $\lambda$ is the instantaneous rate of change of the inclination of $F$ with respect to the inclination of $E$. It is given by the formula

$$
\lambda=\frac{\left(E+2 F v^{\prime}+G v^{\prime 2}\right)\left(\phi \psi_{v^{\prime}}-\psi \phi_{v^{\prime}}\right)}{E \phi^{2}+2 F \phi \psi+G \psi^{2}} .
$$

${ }^{6}$ In space there is, in general, no analogous formula for generalized fields of force. See DeCicco, Extensions of certain dynamical theorems of Halphen and Kasner, Bull. Amer. Math. Soc. vol. 49 (1943) pp. 736-744.

${ }^{7}$ For a generalization to acceleration fields of higher order, see Kasner and Mittleman, $A$ general theorem on the initial curvature of dynamical trajectories, Proc. Nat. Acad. Sci. U.S.A. vol. 28 (1942) pp. 48-52. Also Extended theorems in dynamics, Science vol. 95 (1942) pp. 249-250. Also Kasner and Fialkow, Trans. Amer. Math. Soc. vol. 41 (1937). Fialkow, Initial motion in fields of force, Trans. Amer. Math. Soc. vol. 40 (1936) pp. 495-501. 
For any ordinary positional field of force, $\lambda=0$. However, there are also certain generalized fields of force for which the angular rate $\lambda=0$. These are such that the direction of the force vector at any point 0 does not vary, but its magnitude may.

Since along any line of force $\psi=v^{\prime} \phi$, it is found that along any linealelement $E$ of a line of force, the angular rate $\lambda$ is

$$
\lambda=\frac{\psi_{v^{\prime}}-v^{\prime} \phi_{v^{\prime}}}{\phi} .
$$

Thus the condition that the angular rate $\lambda \neq 1$ is equivalent to stating that there exists a function $v^{\prime}=f(u, v)$ which satisfies the differential equation (15) identically. Thus, in general there are $\infty^{1}$ lines of force.

A rest trajectory is defined as the path described by a particle, constrained to move in the surface $\Sigma$ when acted upon only by a generalized field of force, which starts at rest from a given point $O$.

From the equations (19) together with the initial conditions $\dot{u}=0$, $\dot{v}=0$, it is deduced that a rest trajectory is initially tangent to the line of force through the given point $O$.

Since, on any rest trajectory, there is only one such point $O$ where the particle is at rest, there are $\infty^{2}$ rest trajectories on a surface $\Sigma$.

At the point $O$, let $E_{0}$ be the lineal-element of the line of force. The rest trajectory described by a particle initially at rest at $O$ will be tangent to the line of force along $E_{0}$.

In the present paper, we shall assume that the geodesic curvature of the rest trajectory is not zero at the point $O$. The case of higher order contact with $E_{0}$ will be discussed elsewhere. Since our rest trajectory is tangent to the line of force at $O$, the first derivative must satisfy (15). Therefore from this and (22), it is found that the second derivative $v^{\prime \prime}$ of the rest trajectory at 0 is given by the expression

$$
\begin{aligned}
& \left(\psi_{v^{\prime}}-v^{\prime} \phi_{v^{\prime}}-3 \phi\right) \Delta \\
& \quad+\left(\psi_{v^{\prime}}-v^{\prime} \phi_{v^{\prime}}-\phi\right)\left\{n v^{\prime 3}+(2 m-\nu) v^{\prime 2}+(l-2 \mu) v^{\prime}-\lambda\right\} \\
& \quad+\psi_{u}+\left(\psi_{v}-\phi_{u}\right) v^{\prime}-\phi_{v} v^{\prime 2}=0
\end{aligned}
$$

If $\bar{\Delta}$ denotes the analogous expression (18) for the line of force, we find, by differentiating (15), the following expression

$$
\begin{aligned}
& \left(\psi_{v^{\prime}}-v^{\prime} \phi_{v^{\prime}}-\phi\right) \bar{\Delta} \\
& \quad+\left(\psi_{v^{\prime}}-v^{\prime} \phi_{v^{\prime}}-\phi\right)\left\{n v^{\prime 3}+(2 m-\nu) v^{\prime 2}+(l-2 \mu) v^{\prime}-\lambda\right\} \\
& \quad+\psi_{u}+\left(\psi_{v}-\phi_{u}\right) v^{\prime}-\phi_{v} v^{\prime 2}=0 .
\end{aligned}
$$


Subtracting the above two equations and using the formula (29) wherein $\phi \neq 0$ since the force vector by assumption is not zero, we obtain

$$
(3-\lambda) \Delta=(1-\lambda) \bar{\Delta} \text {. }
$$

As $\rho=\Delta / \bar{\Delta}$ is the ratio of the geodesic curvature of the rest trajectory to that of the line of force, we have proved the following result.

THEOREM 3. The ratio $\rho$ of the geodesic curvatures at $E_{0}$ of the rest trajectory and the line of force is given by the formula

$$
\rho=(1-\lambda) /(3-\lambda) .
$$

By this result, it is seen that if $\rho=1 / 3$, then the angular rate $\lambda=0$ at the lineal-element $E_{0}$.

THEOREM 4. All generalized fields of force for which the ratio of departures $\rho=1 / 3$ must be such that the eliminant with respect to $v^{\prime}$ of the equations

$$
\psi-v^{\prime} \phi=0, \quad \psi_{v^{\prime}}-v^{\prime} \phi_{v^{\prime}}=0,
$$

be identically zero.

This general class of generalized fields of force includes the positional fields as special cases.

ILLINOIS INSTITUTE OF TEChNOLOGY 